\section{A BASIC program for nonparametric post hoc comparisons}

\author{
SUZANNE V. BORYS and JAMES G. CORRIGAN \\ E. R. Johnstone Training and Research Center \\ Bordentown, New Jersey 08505
}

This program is designed to compute post hoc multiple comparisons for a variety of multivariate nonparametric tests, which include: (1) the chi-square $I \times K$ test of homogeneity, (2) the Kruskal-Wallis ANOVA by ranks, (e) Cochran's Q test, and (4) the Friedman test. The procedure presented for these post hoc tests is based on the chi-square analog of Scheffé's theorem (Marascuilo \& McSweeney, 1977). This procedure has the advantage of limiting the probability of committing a Type I error to the level of significance selected regardless of the number of comparisons made. However, Winer (1962) notes that Scheffe's method is the most conservative post hoc test with respect to Type I error and may yield too few significant results when testing for differences between all possible pairs of means. A difference is considered to be significant provided that a zero is not included in the range of the interval that results.

Input. The program begins with the user's selecting the particular test for which the post hoc compari-

\footnotetext{
Development of this program was supported by Grant HD 07329 from the National Institute of Child Health and Human Development.
}

\section{ORPOCO: Orthogonal polynomial coefficients}

\section{DAVID J. WEISS \\ California State University, Los Angeles, California 90032}

ORPOCO extends the usual table of orthogonal polynomial coefficients for trend tests to cover cases with either unequal cell ns or unequal spacing of the levels of the independent variable (or both). The program asks the user for cell sizes and for abscissa values for each cell and then delivers the coefficients for all polynomials up to a user-specified degree. The coefficients are normalized so the user need only square the sum of the products of each cell total and its associated coefficient to get the sum of squares for the component. ORPOCO is a BASIC-PLUS adaptation of an algorithm presented by Emerson (1965).

Limitations. The program is brief ( 84 lines) and need sons are to be done. The user is then asked to supply such information as the number of subjects and the mean rank, proportion, and so on (depending upon the test), for the two groups under comparison, as well as the value of chi square that was used to test the significance of the overall analysis. Questions are presented via a CRT.

Output. A SELECT PRINT command is used to direct the output to a line printer and includes the two means involved in the comparison, the variance of the comparison, the upper and lower limits of the resulting interval, and an asterisk to indicate whether the comparison is significant.

Limitations. The program is limited to making pairwise comparisons only.

Computer and Language. The program is written in BASIC and has been implemented on a WANG System $2200-B$ with $32 \mathrm{~K}$ of memory.

Availability. A program listing may be obtained from Suzanne Borys, Research Department, E. R. Johnstone Training and Research Center, Bordentown, New Jersey 08505.

\section{REFERENCES}

Marascuilo, L. A., \& McSweeney, M. Nonparametric and distribution-free methods for the social sciences. Monterey, Calif: Brooks/Cole, 1977.

WINER, B. J. Statistical principles in experimental design. New York: McGraw-Hill, 1962.

(Accepted for publication November 15, 1980.)

not require large amounts of core, so it should fit in any interactive computer that has a BASIC-PLUS compiler. For example, a problem dimensioned for 100 abscissa values and for a 20-degree polynomial (surely larger than one might need) occupies $10 \mathrm{~K}$ of 16-bit words. Changing the dimensions requires the alteration of only one statement.

Availability. A listing of the program may be obtained without charge from David J. Weiss, Department of Psychology, California State University, Los Angeles, California 90032.

\section{REFERENCE}

EMERSON, P. L. A FORTRAN generator of polynomials orthonormal over unequally spaced and weighted abscissas. Educational and Psychological Measurement, 1965, 25, 867-871.

(Accepted for publication October 25, 1980.) 